\title{
Young women's knowledge and attitudes towards cervical cancer screening at a selected higher education institution in Lesotho: a cross-sectional survey
}

Isabel Nyangu ( $\sim 4321$ bellah@gmail.com )

National University of Lesotho

Tanki Moteane

National University of Lesotho

Research Article

Keywords: cancer, cervical, screening, women, lesotho

Posted Date: November 10th, 2021

DOI: https://doi.org/10.21203/rs.3.rs-1032630/v1

License: (a) This work is licensed under a Creative Commons Attribution 4.0 International License.

Read Full License 


\section{Abstract \\ Background}

In Lesotho, cervical cancer is the most common female cancer and leading cause of death amongst women. In 2019, the annual number of new cancer cases was 477 and 346 women died from cancer related complications in Lesotho. The aim of this study was to assess the knowledge and attitudes of young women towards cervical cancer screening at a selected higher education institution in Lesotho.

\section{Methods}

A quantitative cross-sectional survey design was used to collect data using an interviewer administered semi-structured questionnaire from 80 young women. Random sampling using a fishbowl technique was used to select study respondents. Permission to conduct the study was sought and granted from relevant authorities. Informed consent was sought from the respondents who were identified using codes and participated voluntarily. Data were analysed using Microsoft excel and presented using proportions and diagrams.

\section{Results}

Fifty percent $(n=40)$ of the women did not know cervical cancer screening tests, $70 \%(n=56)$ did not know about the frequency of screening, whilst $75 \%(n=60)$ knew that human papilloma virus (HPV) vaccine is used to prevent cervical cancer. Additionally, $95 \%(n=76)$ had not screened for cervical cancer, $65 \%(n=52)$ perceived cervical cancer screening as painful, 35\% $(n=28)$ said it caused discomfort, and $95 \%(n=76)$ needed more information on cervical cancer screening.

\section{Conclusions}

Even though some respondents had knowledge on cervical cancer and its prevention, the uptake of the screening procedure was low. Negative experiences of pain and discomfort were reported amongst those who had the procedure done. This heightens the need for health education and incorporating regular cervical cancer screening in health care services to increase reassurance, counselling, and the uptake of the procedure amongst young women.

\section{Introduction}

Globally, cervical cancer is the fourth common type of cancer accounting for $6.6 \%$ of all female cancers, [1] with China and India together contributing more than a third of the global cervical cancer burden (106 000 cases and 48000 deaths in China and 97000 cases and 60000 deaths in India),[2]. Cervical cancer is one of the most preventable and curable forms of cancers through early detection and treatment. 
Unfortunately, in many parts of Africa, cervical cancer was previously not identified or treated until it had reached an advanced stage due to insufficient access to health care services, effective screening and early treatment,[3].

Cervical cancer has been steadily increasing in sub-Saharan Africa, with more than 75000 cases and 50 000 deaths yearly, and is further exacerbated by HIV,[4]. In southern Africa, $63.8 \%$ of women with cervical cancer were living with HIV, as were $27 \cdot 4 \%$ of women in eastern Africa,[5]. The highest incidence was reported in Eswatini, with approximately $6.5 \%$ of women developing cervical cancer before the age of 75 years,[2]. The incidence rate of cervical cancer in South Africa was reported between 22.8 and 27 per 100 000 women as compared with the global average of 15.8, and annually there were approximately 6000 new cases reported with 3000 deaths,[6].

With the overall burden of cervical cancer on most African countries expected to surge over the next decades, Lesotho has taken few initiatives to reduce its impact, by introducing free cervical screening program using visual inspection of the cervix with acetic acid (VIA) and Pap test,[7]. In Lesotho, cervical cancer was the leading cause of death among women with an incidence rate of 27.8/100 000 in 2012,[7] and this was attributed to the high HIV incidence and prevalence. Current estimates indicated that every year in Lesotho, 477 women were diagnosed with cervical cancer and 346 died from the disease, particularly in women aged between 40 and 55 years,[7]. Additionally, women present themselves at health centres only when they are sick and this makes it difficult for the health personnel to diagnose the infection at its early and curable stages,[8].

The incidence and prevalence rate of cervical cancer among women in Lesotho continues to increase, with an average of 18 confirmed cases of cervical cancer per month. Despite the availability of free cervical cancer screening services provided in Lesotho, uptake remains limited. This study therefore sought to assess young women's knowledge and attitudes towards cervical cancer screening in Roma, Lesotho

\section{Methodology}

The aim of this study was to assess the knowledge and attitudes of young women enrolled at a selected higher education institution in Lesotho. A quantitative cross-sectional survey was conducted on a sample of 80 young women who were randomly sampled using a fish bowl technique. Eligibility criteria included all female students who were enrolled at the selected higher education institution. A pilot study using 15 respondents who were not included in the study was conducted to ensure validity and reliability of the data collection tool. The questionnaire had three sections which collected information on the respondents' demographics, knowledge, and attitudes towards cervical cancer screening and was found to be adequate in collecting the data. Permission to conduct this study was sought and granted from relevant authorities. Data was collected between the months of June and July 2021. Codes were used to identify the respondents who provided written consent and voluntarily took part in this study. They were allowed to withdraw from the study without any repercussions. They were allowed to ask clarity seeking 
questions before responding to the questions. Upon completion of the study, the final report was availed to the relevant authorities and library at the selected higher education institution.

\section{Patient And Public Involvement}

Young women who responded to the questionnaire were not involved in the design of this study, development of questionnaires, or interpretation of the results. However, they gave consent to provide data on their knowledge and attitudes towards cervical cancer screening.

\section{Results}

\section{Demographic data}

Thirty percent $(n=24)$ of the respondents were aged $18-20$ years, $45 \%(n=36)$ were aged $21-25$ years, $20 \%$ $(n=16)$ were aged $26-30$, and $5 \%(n=4)$ were aged $31-35$ years. Sixty $(75 \%)$ were single, $25 \%(n=20)$ were married, and all of them were sexually active and Christians. Forty-eight $(60 \%)$ were from the rural areas, while $40 \%(n=32)$ were from the urban areas.

\section{Knowledge On Cervical Cancer Screening}

Most of the respondents $(75 \% ; n=60)$ knew that Human Papilloma Virus (HPV) causes cervical cancer. Some of the respondents $(40 \% ; n=32)$, knew that the signs and symptoms of cervical cancer included coital pain, 30\% ( $n=24)$ knew about post-coital bleeding, 20\% ( $n=16)$ knew about post-menopausal bleeding, and only $10 \%(n=8)$ knew that cervical cancer can be asymptomatic. Half $(50 \% ; n=40)$ of the respondents knew about the screening tests for cervical cancer. Some of them $(45 \% ; n=36)$ had information that Pap smear could be used to diagnose cervical cancer, and 5\% $(n=4)$ knew that VIA could be used for screening.

Majority $(70 \% ; n=56)$ of the respondents did not know how often one should screen for cervical cancer, $20 \%(n=16)$ said that an individual should screen every year, and $10 \%(n=8)$ pointed that screening should be done every 3 years. When asked who is eligible for cervical cancer screening, majority $(55 \%$; $n=44)$ of the respondents did not know, 30\% ( $n=24)$ pointed women whose age was greater than 21 years, and 15\% $(n=12)$ pointed that all sexually active women should screen.

Most $(75 \% ; n=60)$ of the respondents suggested that HPV vaccine was the preventive measure against cervical cancer, and $15 \%(n=12)$ pointed that regular screenings are the preventive measure. Figure 1 below illustrates the knowledge in more detail.

\section{Attitudes Towards Cervical Cancer Screening}


Most (95\%: $n=76)$ of the respondents had not yet been screened for cervical cancer, while $5 \%(n=4)$ had screened. Majority $(65 \%: n=52)$ of the respondents thought that the technique used for cervical cancer screening was painful, while $35 \%(n=28)$ said it caused discomfort. Majority ( $80 \%: n=64)$ of the respondents had not screened for cervical cancer due to lack of healthcare facilities in their homes, $15 \%$ $(n=12)$ reported that they had never been asked by a doctor, while $5 \%(n=4)$ reported to have felt no need to screen. Majority $(75 \%: n=60)$ of the respondents preferred to be screened by doctors whilst $25 \%(n=20)$ preferred to be screened by nurses. Majority $(95 \%: n=76)$ of the respondents needed more information on cervical cancer screening.

\section{Discussion}

The age group of 21 to 25 years (45\%) was the largest which is contrary to the age group 26 to 30 years $(50 \%)$ in a study conducted in Niger,[9]. All the respondents were Christians. Engaging religious groups in educating and encouraging their members about cervical cancer screening can also help in reaching women,[10].

Respondents (75\%) knew that HPV was a risk factor of cervical cancer. This is in in line with a study by McBride and Singh,[11] in which more than $70 \%$ of the respondents were aware that HPV could cause cervical cancer but in contrast to a study by Vishwakarma et al.,[12] where only $32 \%$ of subjects knew about HPV. A few of the respondents were aware that cervical cancer can be asymptomatic, present as irregular menstrual bleeding, and present as vaginal bleeding after intercourse. This is less as compared to the study by Vishwakarma et al.,[12] and Urasa and Darj,[13] where more respondents were aware.

Pap smear was the most common screening test known among respondents. This is very similar to a study carried by Owoeye and Ibrahim,[9] in which $44.9 \%$ of the respondents knew about a pap smear test. However, this was very low compared to $88.4 \%, 83 \%$, and $82 \%$ of the respondents that knew in studies by Shah et al.,[14], Mutyaba et al.,[15] and Vishwakarma et al.,[12], respectively. Other screening methods such as cytology-based and HPV DNA which are commonly used in developed countries are not available in Lesotho due to financial and technical constraints.

Cervical cancer screening through Pap smear could be very effective in detecting cervical neoplasia at a primordial stage, and this could be precipitated by a very effective treatment. Only $30 \%$ of the respondents knew that screening should begin at 21 years, which is comparable to $46 \%$ and $54.1 \%$ of respondents that knew in studies by Vishwakarma et al.,[12] and Shah et al.,[14], respectively. Majority of the respondents in this study knew that HPV vaccination was a preventive measure against cervical cancer, and this is a similar finding to a study that reported that $82.7 \%$ of the participants pointed HPV as a preventive measure,[16]. Similarly, majority of the respondents preferred doctors over nurses in performing the Pap smear tests. This is similar to a study by Shekhar et al.,[17] in which $71 \%$ the respondents preferred doctors over nurses, because they perceived cervical cancer screening to be a doctors' procedure. 
The higher mortality of cervical cancer in Lesotho can be attributed to late presentation of the cancer at health facilities. In this study, only $5 \%$ of the respondents had a Pap smear. This is very similar to $5.7 \%$ uptake in Nnewi,[18] and less as compared to $8.3 \%$ among Nigerian women,[19]. Another study conducted in Niger reported an $11.6 \%$ uptake,[9]. In a study carried out in Nigeria, the uptake was $8.7 \%$, [20], and $8.5 \%$ in a study conducted in Ghana,[21]. In contrast, the uptake was $84 \%$ amongst Chinese American women in the United States,[22], whilst more than $80 \%$ of the adult females in United States had a Pap smear during the preceding 3 years,[23]. In majority of developing countries, particularly Lesotho, implementing such measures of coordinated intervention has seemed far impractical.

Poor screening uptake can be attributed to limited healthcare facilities in rural areas which are mostly found in highland regions of the country. In this study, majority of the respondents came from rural areas. This has also been reported in other studies where uneven distribution of medical facilities in the country was the reason for poor uptake,[24], and long distances to such facilities also affected uptake,[16]. In contrast, other reasons for poor cervical cancer screening was due lack of knowledge about the availability of screening, and culturally-influenced reluctance to undergo cervical smear tests,[25].

Respondents who had Pap smears experienced pain (65\%) and discomfort (35\%), which are deemed as misconceptions by other researchers,[26, 27]. The respondents were however keen to obtain more information about cervical cancer screening and this is similar to a study by Imam et al.,[28] where $95 \%$ showed interest in obtaining more information.

\section{Conclusion}

The study shows that the practice of cervical cancer screening is low and there is a high need for information pertaining to cervical cancer screening. Respondents did have some knowledge on cervical cancer, its risk factors, signs and symptoms, and screening tests. They were also aware that the HPV vaccine was a preventative measure against cervical cancer. Their attitudes were positive towards doctors as service providers for cervical cancer screening as compared to nurses.

In contrast, only a few of the respondents had knowledge on when screening could be done and only a few of them had actually had the pap smear procedure done. Of those who actually had the pap smear done, majority had negative experiences of pain and discomfort.

\section{Recommendations}

Health education on cervical cancer screening should be given extensively by the all healthcare professionals to strengthen women's knowledge and encourage them to participate in the uptake of cervical cancer screening. This can be enhanced through various media outlets such as radios and television. Interventions should focus on detailing the nature of the sample and teaching women relaxation techniques so that they can cope with pain and discomfort. Healthcare professionals should only reinforce positive behaviours, so that more women can screen for cervical cancer without fear or any attitude. 


\section{Strengths and limitations}

The study was limited to the knowledge and attitudes of young women on cervical cancer screening.

\section{Declarations}

Ethics approval and Consent to participate: This study and its procedures were approved by the National University of Lesotho Institutional Review Board (NUL-IRB) (ID-201501604) and the Ministry of Health Research and Ethics Committee. All procedures were performed in accordance with relevant guidelines. Written informed consent was obtained from all participants who took part in this study.

Consent to Publish: Not applicable

Availability of Data and Materials: All data generated or analysed during this study are included in this study.

Funding: This study was funded by the National Manpower development Secretariat in Lesotho. Award/grant number not applicable.

Authors contribution: IN and TM both developed and approved the final manuscript.

Acknowledgements. Sincere gratitude to the respondents who took part in this study.

Competing Interests: The authors declare no competing interests

\section{References}

1. Torre L, Islami F, Siegel R, Ward E, Jemal A. Global Cancer in Women: Burden and Trends. Cancer Epidemiology Biomarkers \& Prevention. 2017;26(4):444-457. doi:10.1158/1055-9965.epi-16-0858

2. Arbyn $M$, Weiderpass $E$, Bruni $L$ et al. Estimates of incidence and mortality of cervical cancer in 2018: a worldwide analysis. The Lancet Global Health. 2020;8(2):e191-e203. doi:10.1016/s2214$109 \times(19) 30482-6$

3. World Health Organization (WHO) Africa. Africa advances on goal of cervical cancer elimination across the continent. 2019; From Https://www.afro.who.int/news/africa-advances-goal-cervicalcancer-elimination-across-continent

4. Mboumba Bouassa RS, Prazuck T, Lethu T, et al. Cervical cancer in sub-Saharan Africa: a preventable noncommunicable disease. Expert Rev Anti Infect Ther. 2017;15(6):613-627

5. Stelzle D, Tanaka L, Lee K et al. Estimates of the global burden of cervical cancer associated with HIV. The Lancet Global Health. 2021;9(2):e161-e169. doi:10.1016/s2214-109x(20)30459-9

6. Western Cape Government. Cervical Cancer. 2020; From: https://www.westerncape.gov.za/generalpublication/cervical-cancer 
7. Elizabeth glaser Paediatric AIDS Foundation (EGPAF). 'EGPAF-Lesotho Cervical Cancer Prevention Program. 2018; Available at: https://www.pedaids.org/wpcontent/uploads/2018/12/2018_CaCxFactSheet.pdf.

8. World Health Organization. Framework for Strengthening and Scaling-up of Services for the Management of Invasive Cervical Cancer. Geneva: World Health Organization; 2020; https://apps.who.int/iris. [Accessed 21 December 2020].

9. Owoeye I, Ibrahim. I. Knowledge and attitude towards cervical cancer screening among female students and staff in a tertiary institution in the Niger Delta. International Journal of Medicine and Biomedical Research. 2013;2(1):48-56. doi:10.14194/ijmbr.219

10. Ifediora $C$, Azuike E. Knowledge and attitudes about cervical cancer and its prevention among female secondary school students in Nigeria. Tropical Medicine \& International Health. 2018;23(7):714-723. doi:10.1111/tmi.13070

11. McBride KR, Singh S. Predictors of Adults' Knowledge and Awareness of HPV, HPV-Associated Cancers, and the HPV Vaccine: Implications for Health Education. Health Education \& Behavior. 2018;45(1):68-76. doi:10.1177/1090198117709318

12. Vishwakarma S, Rawat R, Mittal N, Shree P. Knowledge, attitude and practices about cervical cancer screening among nursing staff in rural tertiary care center. Int J Reprod Contracept Obstet Gynecol. 2018;7(9):3796. doi:10.18203/2320-1770.ijrcog20183797

13. Urasa M,Darj E. Knowledge of cervical cancer and screening practices of nurses at a regional hospital in Tanzania. African Health Sciences 2011;11(1);48-57. doi: 10.4314/ahs.v11i1.64992.

14. Shah, V. et al. Awareness and knowledge of cervical cancer and its prevention among the nursing staff of a tertiary health institute in Ahmedabad, Gujarat, India', Ecancermedicalscience. 2012;6(1); 1-6. doi: 10.3332/ecancer.2012.270.

15. Mutyaba T, Mmiro F, Weiderpass E. Knowledge, attitudes and practices on cervical cancer screening among the medical workers of Mulago Hospital, Uganda. BMC Med Educ. 2006;6(1). doi:10.1186/1472-6920-6-13

16. Nyamambi E, Murendo C, Sibanda N, Mazinyane S. Knowledge, attitudes and barriers of cervical cancer screening among women in Chegutu rural district of Zimbabwe. Cogent Soc Sci. 2020;6(1):1766784. doi:10.1080/23311886.2020.1766784

17. Shekhar S, Sharma C, Thakur S, Raina N. Cervical Cancer Screening: Knowledge, Attitude and Practices among Nursing Staff in a Tertiary Level Teaching Institution of Rural India. Asian Pacific Journal of Cancer Prevention. 2013;14(6):3641-3645. doi:10.7314/apjcp.2013.14.6.3641

18. Udigwe GO. Knowledge, attitude and practice of cervical cancer screening (pap smear) among female nurses in Nnewi, South Eastern Nigeria. Nigerian journal of clinical practice. 2006;40-43. doi: 10.4314/njcp.v9i1.11267.

19. Ayinde OA, Omigbodun AO, llesanmi AO. Awareness of cervical cancer, Papanicolaou's smear and its utilisation among female undergraduates in Ibadan. Afr J Reprod Health. 2004;8(3):68-80. PMID: 17348326. 
20. Adefuye P. Knowledge and practice of cervical cancer screening among female professional health workers in a sub-urban district of Nigeria. Nigerian Medical Practitioner. 2006;50(1). doi:10.4314/nmp.v50i1.28829

21. Adanu R. Cervical Cancer Knowledge and Screening in Accra, Ghana. J Womens Health Gend Based Med. 2002;11(6):487-488. doi:10.1089/152460902760277822

22. Lee-Lin F, Pett M, Menon U et al. Cervical Cancer Beliefs and Pap Test Screening Practices Among Chinese American Immigrants. Oncol Nurs Forum. 2007;34(6):1203-1209. doi:10.1188/07.onf.12031209

23. Hewitt M, Devesa SS, Breen N. Cervical cancer screening among U.S. women: analyses of the 2000 National Health Interview Survey. Prev Med. 2004;39:270-8.

24. Cheah PL, Looi LM. Carcinoma of the uterine cervix: a review of its pathology and commentary on the problem in Malaysians. Malays J Pathol. 1999; 21, 1-15

25. . Wong L, Wong Y, Low W, Khoo E, Shuib R. Cervical cancer screening attitudes and beliefs of malaysian women who have never had a pap smear: A qualitative study. Int J Behav Med. 2008;15(4):289-292. doi:10.1080/10705500802365490

26. Denic S, Bener A. Consanguinity decreases risk of breast cancer - cervical cancer unaffected. Br J Cancer. 2001;85: 1675-1679. https://doi.org/10.1054/bjoc.2001.2131

27. Gamarra CJ, Paz EPA and Griep RH. Knowledge, attitudes and practice related to Papanicolaou smear test among Argentina's women. Revista de Saude Publica. 2005;39(2): 270-276. doi: 10.1590/s0034-89102005000200019.

28. Imam SZ, et al. Perceptions and practices of a Pakistani population regarding cervical cancer screening. Asian Pacific Journal of Cancer Prevention. 2008;9(1):42-44.

\section{Figures}




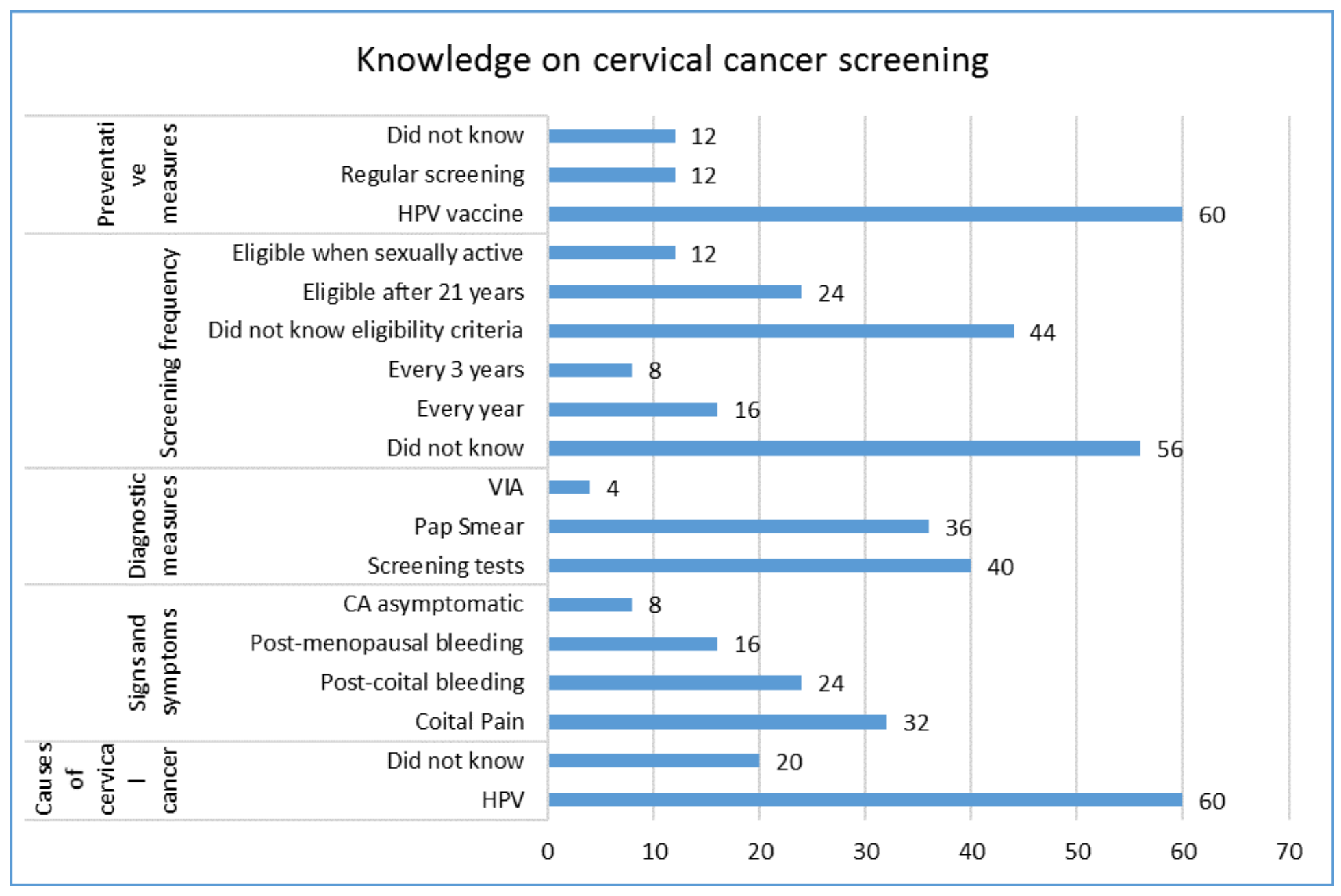

\section{Figure 1}

Knowledge on cervical cancer screening 